Case Report

\title{
Pandemics 2020 -COVID 19 Silent Hypoxia and Polysubstance Use: A Unique Case Report
}

\author{
Michael D. Owens *, Megan Halliday*
}

Naval Medical Center Portsmouth, Portsmouth, Virginia; E-Mails: Michael.d.owens18.civ@mail.mil; megan.h.halliday.mil@mail.mil

* Correspondence: Michael D. Owens and Megan Halliday; E-Mails: Michael.d.owens18.civ@mail.mil; megan.h.halliday.mil@mail.mil

Academic Editor: Gerhard Litscher

OBM Integrative and Complementary Medicine 2021, volume 6 , issue 3

doi:10.21926/obm.icm. 2103025
Received: December 24, 2020

Accepted: August 19, 2021

Published: August 30, 2021

\begin{abstract}
The coronavirus (COVID-19) pandemic continues to bring challenges in identification and management of which the "happy hypoxic" or silent hypoxia presentation has been found to present its own unique challenge. A 67-year-old African American COVID-19 positive female presents to the emergency department (ED) with a chief complaint of decreased oral intake and responsiveness, oriented and stable, with oxygen saturations found to drop as low as $47 \%$. The patient was successfully managed with supplemental oxygen and determined to be a "happy hypoxic" with recent polysubstance abuse. Silent hypoxia or the "happy hypoxic" patient is another unique phenomenon associated with the variable presentations of COVID19. Multiple etiologies and pathophysiologic explanations exist to explain this stable patient whom would otherwise be expected to require aggressive management with a poor prognosis. An alternative diagnosis should be explored for those silent hypoxic patients with symptoms.
\end{abstract}

\section{Keywords}

COVID 19; silent hypoxia; happy hypoxia; opioids; case report

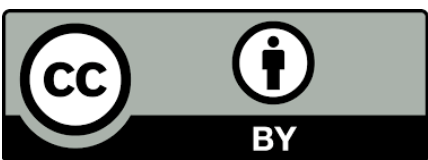

(C) 2021 by the author. This is an open access article distributed under the conditions of the Creative Commons by Attribution License, which permits unrestricted use, distribution, and reproduction in any medium or format, provided the original work is correctly cited. 


\section{Introduction}

The coronavirus (COVID-19) pandemic continues to strain medical resources, facilities, and national infrastructure throughout the world. As of July 20, 2021 the World Health Organization (WHO) reported over 190 million cases with over 4 million deaths worldwide [1]. One unique challenge identified with this novel outbreak is the stable hypoxic patient also referred to as "happy hypoxia." Current studies have shown that hypoxemia in positive COVID-19 patients is independently associated with in-hospital mortality. These studies show that $14 \%$ of associated pneumonia cases are severe, and $5 \%$ require intensive care with approximately two-thirds mortality rate [2].

A surprising finding in some patients with COVID-19 is the "happy hypoxic" phenomenon. Severe COVID-19 cases tend to struggle with breathing, present with dyspnea and chest complaints, and have associated saturation levels on pulse oximetry that are fairly consistent with their clinical presentation. The "happy hypoxic" patient presenting with potentially fatal low saturations levels with no or minimal symptoms is a diagnostic and management challenge $[3,4]$.

\section{Case Report}

A 67-year-old African-American female with a significant past medical history a history of heart failure with preserved ejection fraction, type 2 diabetes, hypertension, and obstructive sleep apnea (OSA) arrived via emergency medical services (EMS) for a chief complaint of decreased oral intake. Per the patient's family she is normally independent, active, and oriented, and has no history explaining this change. The patient reported a recent positive COVID-19 test days prior and decreased oral intake. This COVID test was obtained by the patient due to a recent positive exposure (husband) with associated mild respiratory symptoms, including cough. The family stated that prior to calling EMS she was feeling cold and weak and attributed these symptoms to a decrease in oral intake, especially fluids. On a review of systems (ROS) assessment the patient denied fever, headache, chest pain, difficulty breathing, sore throat, abdominal pain, nausea or vomiting, or diarrhea. She did endorse an occasional cough.

In the emergency department (ED) the patient's triage vital signs were: temperature $99^{\circ} \mathrm{F}$ $\left(37.2^{\circ} \mathrm{C}\right)$, heart rate 91 , respiratory rate 14 , and room air saturation of $47 \%$. An immediate accucheck was recorded as 118 milligrams per deciliter (mg/dL) (reference range: 70-105 mg/dL). The physical exam noted a female in no distress found to be alert and oriented x3. Her HEENT, heart, lung, abdominal, skin, and extremity exam did not note any significant abnormalities. A thorough neurological exam found a cooperative patient with a Glasgow Coma Score (GCS) of 15; alert and oriented; with no associated speech, sensory, or motor deficits appreciated. After the patient was placed on a monitor and an oxygen saturation was observed at $47 \%$ with the nurse recording an "excellent pleth". She immediately improved to $100 \%$ on $10 \mathrm{~L}$ nonrebreather (NRB). The patient showed no signs of distress, increase respiratory rate, or change in mental status associated with this low saturation recording.

The patient was kept on the NRB 10L with saturations remaining at 97\%-100\%. An attempt was made to wean her from oxygen as a monitor or other recording error was considered. After approximately 5 minutes on room air the patient's saturation levels declined to the 70s during which an arterial blood gas ( $A B G$ ) was obtained that correlated with the monitor. The ABG finding results 
confirming this monitor reading were $\mathrm{pH} 7.38$ (reference range: $7.35-7.45$ ), $\mathrm{PaO}_{2} 42 \mathrm{mmHg}$ (reference range: $75-100 \mathrm{mmHg}$ ), $\mathrm{PaCO}_{2} 48 \mathrm{mmHg}$ (reference range: $35-46 \mathrm{mmHg}$ ), $\mathrm{HCO}_{3} 38 \mathrm{meq} / \mathrm{L}$ (reference range: $22-26 \mathrm{meq} / \mathrm{L}$ ), $\mathrm{SaO}_{2} 76 \%$ (reference range $95-100 \%$ ). No symptoms or mental status changes were identified during this observed desaturation. The patient was put back on the NRB and remained at 97\%-100\%. Multiple labs were ordered and a chest $x$-ray (CXR) and ECG were obtained. Notable abnormalities included a creatinine level of 1.45 milligrams per deciliter $(\mathrm{mg} / \mathrm{dL}$ ) (reference range: $0.5-1.0 \mathrm{mg} / \mathrm{dL}$ ), BUN of 36 milligrams per deciliter $(\mathrm{mg} / \mathrm{dL}$ ) (reference range: 7-17 $\mathrm{mg} / \mathrm{dL}$ ), CO2 of 39 millimoles per liter ( $\mathrm{mmol} / \mathrm{L}$ ) (reference range: 22-30 mmol/L), AST 51 units per liter (U/L) (reference range 14-36 U/L), LDH 873 units per liter (U/L) (reference range: 313-618 U/L), c-reactive protein 155.1 milligrams per liter $(\mathrm{mg} / \mathrm{L}$ ) (reference range: 0-9.9 mg/L), d-dimer 0.66 micrograms per milliliter (UG/mL) (reference range 0.27-0.50 UG/mL), fibrinogen 660 milligrams per deciliter (mg/dL) (reference range: $225-456 \mathrm{mg} / \mathrm{dL}$ ) , GGT 337 units per liter (U/L) (reference range: 12-43 U/L), absolute lymphocytes $0.95 \times 10^{3}$ per microliter $(/ \mathrm{mcL}$ ) (reference range 1.00 $3.50 \times 10^{3} / \mathrm{mcL}$ ), and a urine drug screen positive for cocaine and opiates. The complete blood count $(C B C)$ hemoglobin $12.2 \mathrm{~g} / \mathrm{dL}$ (reference range: $11.5-15.5 \mathrm{~g} / \mathrm{dL}$ ) and hematocrit $37.9 \%$ (reference range 34.0-48.0\%) were normal.

The CXR was read by radiology as: 1) prominence and indistinctness of pulmonary vasculature and 2) subtle peripheral hazy opacities that can be seen in the setting of Covid pneumonia. The ECG noted a normal sinus rhythm at 88 beats per minute, with normal intervals, and no acute ST abnormalities.

In the ED the patient was given a gentle $500 \mathrm{~mL}$ normal saline fluid bolus for suspected acute kidney injury (AKI) and transferred to the intensive care unit (ICU). During her ICU stay, the patient admitted to a history or polysubstance abuse that included heroin, alcohol, and cocaine with recent use. She was quickly weaned from the NRB to nasal cannula and transferred to the floor. The patient was started on Decadron, Lovenox, and remdesivir and removed from oxygen. On hospital day 4, the patient was discharged without an oxygen requirement and any physical complaints.

\section{Discussion}

Normal oxygen levels of $95 \%$ or higher is to be expected in patients presenting without respiratory complaints. Current management strategies for respiratory complaints include providing supplemental oxygen to patients who fall below $94 \%$. Studies have validated that sex, age, comorbidities, and certain inflammatory biomarkers are associated with an increased mortality from COVID [2]. Dyspnea as well as pulse oximetry $\left(\mathrm{SpO}_{2}\right)$ independent of age and sex have been

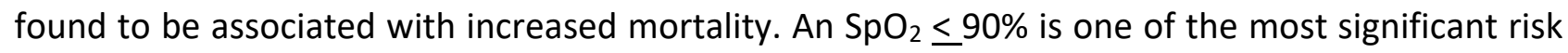
factors for fatal outcomes in COVID-19 patients [2]. Severely hypoxic patients without symptoms of dyspnea present a different clinical picture. This silent hypoxia has been seen to occur in patients with atelectasis, intrapulmonary shunt, or right-to-left intracardiac shunt [5].

The typical COVID-19 patient in respiratory distress requiring interventions does not present with the prominent dyspnea and lung stiffness associated with hypoxia seen in typical acute respiratory distress syndrome [6]. Gattinoni et al. suspect the etiology of this hypoxia in compliant lungs is due to a loss of lung perfusion regulation and hypoxic vasoconstriction.

A pulmonary emboli (PE) theory proposes that the "small blood vessels of the lung are being showered with clots" creating a shunt and should be treated with heparin [3]. If the d-dimer level is 
elevated the heparin dose is increased. Another shunt explanation proposed is a probe-patent foramen ovale based on autopsies at the Mayo Clinic that found an overall incidence of foramen ovale of $27.3 \%$ in normal hearts [4, 7]. It is theorized that COVID-19 is revealing these patients. However, hypoxia in this case should not improve with oxygen supplementation.

Additional explanations include a leftward oxyhemoglobin dissociation curve shift due to increased heme levels with harmful iron levels $\left(\mathrm{Fe}^{3+}\right)$; intrapulmonary shunting caused by lung edema, loss of surfactant, or alveolar collapse causing non-aerated lung to be perfused; loss of lung perfusion regulation caused by pulmonary vasoconstriction triggered by an inflammatory process; or that impaired diffusion capacity may occur from debris covering the lung basement membrane [5].

Pneumolysis, alveolar-capillary destruction, leading to hypoxemia, hypercapnia, and pulmonary shunting may be an additional contributing factor that carries three pathophysiological stages. These three stages are proposed to be: 1) Silent Hypoxemia, 2) Gasping, and finally 3) Death zone. This progressive pneumolysis in association with inflammation, overexpressed immunity, and the high-altitude pulmonary edema (HAPE) type of reaction results in pulmonary shunting leading to the findings seen in silent hypoxemia [8]. Gustavo Zubieta-Callga et al. argues that COVID-19 hypoxia is similar to high-altitude illness in pathophysiology and severity and can be potentially managed using erythropoietin (EPO), acetylsalicylic-acid, anti-inflammatories, oxygen, anitussives, and antibiotics while avoiding counterproductive ventilation [9].

Tobin et al. propose that the virus may have an idiosyncratic effect on the respiratory control system via angiotensin-converting enzyme 2 (ACE 2) receptors in the carotid body [10]. Elderly and diabetics sometimes exhibit a blunting of this reflex and are prone to silent hypoxia. Opioids are also known to blunt this reflex. This patient is elderly, type 2 diabetic, with recent opioid substance abuse all of which may impact respiratory control. Angiotensin-converting enzyme 2 (ACE2) sex-related activity has shown worse outcomes in men versus women but may be due to a higher prevalence of hypertension and heart failure among men and requires additional study [11].

This complex immunologic and hematologic pathophysiology not only impacts the respiratory system, but also is readily known to have multisystem organ impacts. These impacts include coagulation disorders, cardiovascular disorders such as acute cardiac injury, acute liver and kidney injury, and neurological manifestations such as sensory loss of taste and smell to seizures and Guillian-Barré syndrome [12]. This patient was noted to have some multisystem laboratory changes to include the kidney, liver, and coagulation.

This patient's decreased responsiveness to verbal stimuli presentation was felt to be due to her polysubstance abuse and decreased oral intake versus hypoxia. The patient did not complain nor was she observed to exhibit any respiratory or cardiovascular symptoms. Her presentation appeared to correct consistent with her polysubstances ingested.

This case provides an insight into the recently identified COVID-19 phenomenon of the "happy hypoxic" or stable hypoxic patient. Studies have shown that this patient based on age, sex, demographics, past medical history, social history, and room air oxygen saturation level has a high mortality rate. Stable hypoxic COVID-19 patients are a unique subset of patients requiring a deeper understanding of presentation and management. This case report illustrates the need to remain vigilant in the history and physical assessment with a patient presenting with COVID-19 silent hypoxia and polysubstance abuse. 


\section{Conclusion}

Silent hypoxia or "happy hypoxia" is a clinical presentation when a positive COVID-19 patient exhibits potentially fatally low oxygen saturation levels without symptoms or associated pathology. While a variety of associated theories may explain this phenomenon, the exact pathophysiology and cause for these presentations is uncertain. This case is a presentation of a positive COVID-19 patient presenting with stable silent hypoxia and polysubstance abuse with an unremarkable inpatient hospital course.

\section{Author Contributions}

Michael Owens contributions include writing the majority of the paper, reference research, and edits. Megan Holliday assisted with additional reference searches (2 references), laboratory findings, clinical presentation writing and edits, and final grammar as well as edits for clarity.

\section{Competing Interests}

The authors have declared that no competing interests exist.

\section{References}

1. Coronavirus disease (COVID-19) weekly epidemiological update and weekly operational update [Internet]. World Health Organization; 2021. Available from: http://who.int/emergencies/diseases/novel-coronavirus-2019/situation-reports/.

2. Xie J, Covassin N, Fan Z, Singh P, Gao W, Li G, et al. Association between hypoxia and mortality in patients with COVID-19. Mayo Clin Proc. 2020; 95: 1138-1147.

3. Couzin-Frankel J. The mystery of the pandemic's 'happy hypoxia'. Science. 2020; 368: 455-456.

4. Fisher HK. Hypoxemia in COVID-19 patients: An hypothesis. Med Hypotheses. 2020; 143: 1-4.

5. Dhont S, Derom E, Van Braeckel E, Depuydt P, Lambrecht BN. The pathophysiology of 'happy' hypoxemia in COVID-19. Respir Res. 2020; 21: 1-9.

6. Gattinoni L, Coppola S, Cressoni M, Busana M, Rossi S, Chiumello D. COVID-19 does not lead to a "typical" acute respiratory distress syndrome. Am J Respir Crit Care Med. 2020; 201: 12991300.

7. Hagen PT, Scholz DG, Edwards WD. Incidence and size of patent foramen ovale during the first 10 decades of life: An autopsy study of 965 normal hearts. Mayo Clin Proc. 1984; 59: 17-20.

8. Zubieta-Calleja G, Zubieta-DuUrioste N. Pneumolysis and "silent hypoxemia" in COVID-19. Indian J Clin Biochem. 2020; 36: 1-5.

9. Zubieta-Calleja G, Zubieta-DeUrioste N, Venkatesh T, Das KK, Soliz J. COVID-19 and pneumolysis simulating extreme high-altitude exposure with altered oxygen transport physiology; multiple diseases, and scarce need of ventilators: Andean Condor's-eye-view. Rev Recent Clin Trials. 2020; 15: 347-359.

10. Tobin MJ, Laghi F, Jubran A. Why COVID-19 silent hypoxemia is baffling to physicians. Am J Respir Crit Care Med. 2020; 202: 356-360. 
11. Salah HM, Mehta JL. Hypothesis: Sex-related differences in ACE2 activity may contribute to higher mortality in men versus women with COVID-19. J Cardovasc Pharmacol Ther. 2021; 26: 114-118.

12. Robba C, Battaglini D, Pelosi P, Rocco PR. Multiple organ dysfunction in SARS-CoV-2: MODSCoV-2. Expert Rev Respir Med. 2020; 14: 865-868.

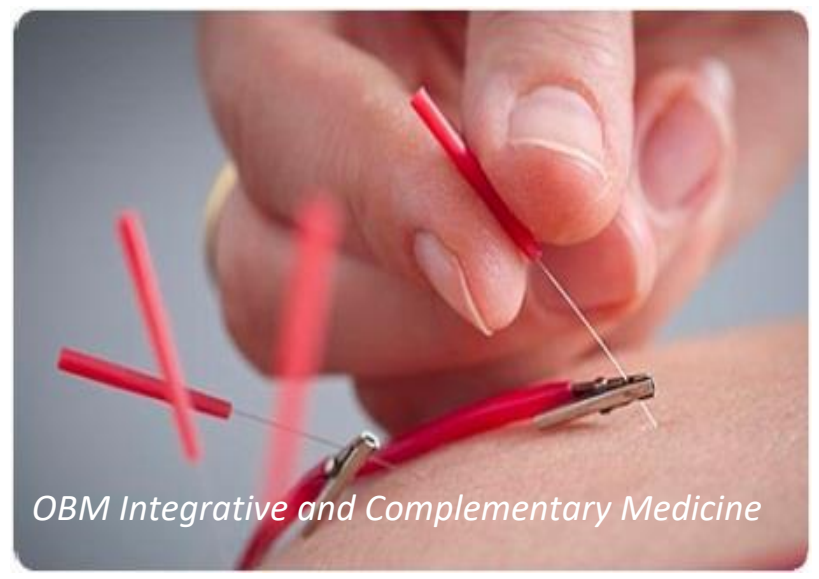

Enjoy $O B M$ Integrative and Complementary Medicine by:

1. Submitting a manuscript

2. Joining in volunteer reviewer bank

3. Joining Editorial Board

4. Guest editing a special issue

For more details, please visit:

http://www.lidsen.com/journals/icm 